PROCEEDINGS OF THE

AMERICAN MATHEMATICAL SOCIETY

Volume 129, Number 1, Pages 247-256

S 0002-9939(00)05527-1

Article electronically published on July 27, 2000

\title{
POSITIVE AND NEGATIVE 3-K-CONTACT STRUCTURES
}

\author{
WŁODZIMIERZ JELONEK \\ (Communicated by Christopher Croke)
}

\begin{abstract}
The aim of this paper is to give a characterization of 3-K-contact and quasi 3-K-contact manifolds.
\end{abstract}

\section{INTRODUCTION}

The aim of this paper is to describe the class of Riemannian manifolds that is closely related to quaternionic-Kähler manifolds. It is well known that there is a close connection between the quaternionic-Kähler manifolds with positive scalar curvature and the class of 3-Sasakian manifolds (see [K], [Ku, [I1, [BGM]). Ishihara [11] has shown that every fibered Riemannian 3-Sasakian bundle has a base which is a quaternionic-Kähler manifold with positive scalar curvature. The 3Sasakian manifolds are essentially the $S O(3)$-principal bundles (or principal orbifold bundles) over quaternionic-Kähler manifolds (see BGM]). We have proved in [J2] that for any quaternionic-Kähler manifold $M$ with negative scalar curvature there exists an $S O(3)$-principal fibre bundle $P$ such that $P$ is an $\mathcal{A}$-manifold, the projection $p: P \rightarrow M$ is a Riemannian submersion and $P$ admits three $\mathrm{K}$ contact structures satisfying the relations very similar to those characterizing the 3 -K-contact structures. We have called such structures the quasi 3-K-contact structures. Recently similar structures were introduced by S. Tanno ([T]). S. Tanno introduced $n S$-structures (an $n S$-structure is a quasi 3-K-contact structure satisfying an additional condition analogous to that characterizing the 3-Sasakian structure in the positive case). S. Tanno proved that every quaternionic-Kähler manifold with negative scalar curvature admits an $S O(3)$-principal fibre bundle $P$ with canonical $n S$-structure and that any 3 -K-contact structure on a 7 -dimensional manifold has to be 3 -Sasakian (respectively any quasi 3-K-contact structure on a 7-dimensional manifold has to be an $n S$-structure). In the present paper we extend these results to any dimension $4 n+3>11$. The work is devoted to the study of general quasi 3 -K-contact structures. We shall also call them negative 3 -K-contact structures and the usual 3-K-contact structure we shall call positive 3-K-contact structure. The manifold $P$ with 3 -K-contact (positive or negative) structure has dimension $4 n+3$. We show that if $n \neq 2$, then every (positive) 3 -K-contact structure is 3-Sasakian and every negative 3 -K-contact manifold is an $\mathcal{A}$-manifold whose Ricci tensor has two constant eigenvalues.

Received by the editors May 18, 1998 and, in revised form, March 26, 1999.

1991 Mathematics Subject Classification. Primary 53C25, 53C15.

Key words and phrases. 3-K-contact structure, quaternionic-Kähler manifold, Sasakian structure, $\mathcal{A}$-manifold.

(C)2000 American Mathematical Society 


\section{Preliminaries}

We start by recalling some basic facts concerning the quaternion-Kähler geometry (see $[\mathrm{S}], \mathrm{Sw},[\mathrm{B}], \mathrm{GL}]$ ) and K-contact structures. By $\mathfrak{X}(M)$ we denote the Lie algebra of all local vector fields on $M$. If $D$ is a vector bundle over $M$, then by $\Gamma(D)$ we denote the set of all local sections of $D$. We also write $\mathcal{A}^{k}(M)=\Gamma\left(\wedge^{k} T M^{*}\right)$. Let $(M, g)$ be an oriented Riemannian manifold, let $\operatorname{dim} M=4 n$ and let $S O(M)$ be the $S O(4 n)$-principal fibre bundle of oriented orthonormal frames $u: \mathbb{R}^{4 n} \rightarrow T M$. By $\nabla$ we denote the Levi-Civita connection of $(M, g)$. Let $\mathcal{G} \subset \operatorname{End}(T M)$ be the 3-dimensional subbundle locally generated by three almost complex structures $\{I, J, K\}$ compatible with the metric $g$ and satisfying the additional condition $I \circ J=-J \circ I=K$. If $n>1$, then a manifold $(M, g)$ with the bundle $\mathcal{G}$ satisfying the above conditions is called the almost quaternion Hermitian manifold. The subbundle $\mathcal{G}$ is called parallel (with respect to $\nabla$ ) if for every section $A \in \Gamma(\mathcal{G})$ and for every $X \in \mathfrak{X}(M)$ we have $\nabla_{X} A \in \mathcal{G}$. An almost quaternion Hermitian manifold $(M, g)$ with a parallel bundle $\mathcal{G}$ is called a quaternionic-Kähler manifold. If $\operatorname{dim} M=4$, then we shall call $(M, g)$ a quaternionic-Kähler manifold if it is antiself-dual and Einstein (we shall always choose an orientation of $M$ in such a way that $\left.\mathcal{G}=\bigwedge^{+} M\right)$. Every quaternionic-Kähler manifold is an Einstein manifold. Let $(M, g)$ be a Riemannian manifold and $\xi$ be a unit Killing vector field on $M$. Let us define a tensor field $\phi$ by $\phi(X)=\nabla_{X} \xi$ and a 1 -form $\eta(X):=g(\xi, X)$. Then we call $(M, g, \xi, \phi, \eta)$ a $\mathrm{K}$-contact structure if the following relation is satisfied:

$$
\phi^{2}=-i d+\eta \otimes \xi
$$

Let us assume that $\xi_{0}$ is a Killing vector field of constant length on $M$. We shall find the conditions under which the Killing vector field $\xi=c \xi_{0}$ where $c=\frac{1}{\left\|\xi_{0}\right\|}$ defines the K-contact metric structure. Let us denote by

$$
H=k e r \eta=\{X: g(\xi, X)=0\}
$$

the distribution of horizontal vectors on $M$. The following Lemma is well known (see [J1]).

Lemma. Under the above assumptions the Killing vector field $\xi$ gives the K-contact structure on $M$ if and only if the tensor $J=\phi_{\mid H}$ is the almost complex structure on the bundle $H$, i.e. $J^{2}=-i d_{\mid H}$.

The mapping $p: P \rightarrow M$ is a Riemannian submersion (see [ON]) if for every $y \in P$ the mapping $d_{y} p: H_{y} \rightarrow T_{x} M$ is an isometry, where $x=p(y)$ and $H_{y}$ is an orthogonal complement of the vertical space $V_{y}=T_{y} F_{x}$ where $F_{x}=p^{-1}(x)$. In the sequel we shall use the O'Neill's tensors $T, A$. They are defined as follows:

$$
\begin{aligned}
A_{X} Y & =\mathcal{V}\left(\nabla_{\mathcal{H} X} \mathcal{H} Y\right)+\mathcal{H}\left(\nabla_{\mathcal{H} X} \mathcal{V} Y\right) \\
T_{X} Y & =\mathcal{H}\left(\nabla_{\mathcal{V} X} \mathcal{V} Y\right)+\mathcal{V}\left(\nabla_{\mathcal{V} X} \mathcal{H} Y\right)
\end{aligned}
$$

where $\mathcal{H}, \mathcal{V}$ respectively denote the projections on the horizontal and vertical subbundles $H, V$ of $T P=H \oplus V$. Finally, let us recall that a Riemannian manifold $(M, g)$ is called an $\mathcal{A}$-manifold (see $\mathrm{G}]$ ) (we shall write $M \in \mathcal{A}$ in such a case) if the Ricci tensor of $(M, g)$ satisfies the condition $\nabla_{X} \rho(X, X)=0$ for all local vector fields $X \in \mathfrak{X}(M)$. 


\section{Quasi 3-K-COntact STRUCtures}

We start with a definition of the 3-K-contact and quasi 3-K-contact structure.

Definition. Let $(P, g)$ be a Riemannian manifold that admits three distinct $\mathrm{K}$ contact structures $\left(\phi_{i}, \xi_{i}, \eta_{i}\right)$ such that

$$
\text { (a) } g\left(\xi_{i}, \xi_{j}\right)=\delta_{i j}, \quad(b)\left[\xi_{i}, \xi_{j}\right]=2 \epsilon_{i j k} \xi_{k}, \quad(c) \phi_{i} \xi_{j}=-\epsilon_{i j k} \xi_{k}
$$

where $\phi_{i}=\nabla \xi_{i}, \eta_{i}(X)=g\left(\xi_{i}, X\right)$. Let us denote by $H$ the horizontal distribution $H=\operatorname{ker} \eta_{1} \cap \operatorname{ker} \eta_{2} \cap \operatorname{ker} \eta_{3}=\bigcap$ ker $\eta_{i}$ and let us define the almost complex structures $J_{i}$ on $H$ by the formulas $J_{i}=-\phi_{i \mid H}$. We shall call $\left(P, \xi_{1}, \xi_{2}, \xi_{3}\right)$ the 3 -K-contact structure (or positive 3 -K-contact structure) if (for $i \neq j$ )

$$
J_{i} \circ J_{j}=\epsilon_{i j k} J_{k}
$$

and the quasi 3 -K-contact structure (or negative 3 -K-contact structure) if $(i \neq j)$

$$
J_{i} \circ J_{j}=-\epsilon_{i j k} J_{k}
$$

The Riemannian manifold $(P, g)$ with positive (negative) 3-K-contact structure we shall call positive (negative) 3 -K-contact manifold.

By $V=\operatorname{span}_{\mathbb{R}}\left\{\xi_{1}, \xi_{2}, \xi_{3}\right\}$ we shall denote the vertical bundle of $P$. It is clear that $T P=H \oplus V$ and $H \perp V$. The distribution $V$ is integrable and the leaves are totally geodesic submanifolds of $P$. If $P$ is complete, then the leaves are 3 dimensional spherical space forms.

Remark. Our definition of positive 3-K-contact structure is equivalent to the usual one (see [Ku], JJ1]). S. Tanno defined in [T] an $n S$-structure. The $n S$-structure is a negative 3 -K-contact structure (condition (6.3) in [T] is equivalent to $J_{i} \circ J_{j}=$ $\left.-\epsilon_{i j k} J_{k}\right)$ satisfying an additional condition $((6.4)$ in $[\mathrm{T}])$. We shall show in the sequel that if $\operatorname{dim} P=4 n+3 \neq 11$, then every negative 3 -K-contact structure must be an $n S$-structure.

Note that if $P$ is complete, then it admits an action of the group $S U(2)$ or $S O(3)$ of isometries of $P$. Let us assume that $(P, g)$ is a fibre bundle $p: P \rightarrow M$ and the group $G(G=S U(2)$ or $G=S O(3))$ acts on $P$ on the right by isometries such that the orbits of the action coincides with the fibers of $p$, i.e. $p^{-1}(p(x))=\operatorname{orb}_{G}(x)$ and $M=P / G$. Let us assume that Killing tensors $\xi_{1}, \xi_{2}, \xi_{3}$ corresponding to the basis of the Lie algebra $\mathfrak{g}=\mathfrak{s o}(3)$ of $G$ define on $P$ the (positive or negative) 3 -K-contact structure. Thus the fibers are totally geodesic submanifolds of $P$ isometric to $G / \Gamma$ where $\Gamma$ is a discrete subgroup of $G$. If $\Gamma=\{e\}$, i.e. $p: P \rightarrow M$ is a $G$-principal fibre bundle over $M$, then we shall call $P$ the 3 -K-contact principal fibre bundle. If $X \in \mathfrak{X}(M)$, then by $X^{*} \in \Gamma(H)$ we mean a horizontal lift of $X$, i.e. the horizontal vector field $X^{*} \in \mathfrak{X}(P)$ which is $p$ related with $X\left(d p\left(X^{*}\right)=X \circ p\right)$.

Theorem 1. Let $(P, g)$ be an $S O(3)$ principal 3-K-contact bundle (positive or negative) over a manifold $M$. Then the metric $g$ induces a metric $g_{*}$ on $M$ such that $p:(P, g) \rightarrow\left(M, g_{*}\right)$ is a Riemannian submersion and $\left(M, g_{*}\right)$ is an almost quaternion Hermitian manifold.

Proof (Compare [I1]). We start by constructing a bundle $\mathcal{G} \subset \operatorname{End}(T M)$ locally spanned by three almost complex structures giving the quaternionic structure on 
$M$. Let $\sigma \in \Gamma(P)$ be a local section of the bundle $P$. We shall define on $U=\operatorname{dom} \sigma$ three almost complex structures

$$
J_{i}^{\sigma}=\epsilon d p \circ \phi_{i} \circ \sigma^{*}
$$

where $\sigma^{*}(X)(x)=X_{\sigma(x)}^{*}, \epsilon=-1$ if $(P, g)$ is a positive 3 -K-contact manifold, $\epsilon=1$ if $(P, g)$ is a negative manifold and $X^{*}$ denotes the horizontal lift of the field $X \in$ $\mathfrak{X}(M)$. We have $d p \phi_{i} d \sigma(X)=d p \circ \phi_{i}\left(d \sigma(X)-\sigma^{*}(X)+\sigma^{*}(X)\right)=d p \circ \phi_{i} \sigma^{*}(X)$ since $d \sigma(X)-\sigma^{*}(X) \in \Gamma(V)$ and $\phi_{i}(V) \subset V$. Thus in fact we have

$$
J_{i}^{\sigma}=\epsilon d p \circ \phi_{i} \circ d \sigma
$$

It is obvious from the definition of positive (negative) 3 -K-contact structure that the almost complex structures $\left\{J_{1}^{\sigma}, J_{2}^{\sigma}, J_{3}^{\sigma}\right\}$ define on $U$ the almost quaternionic structure, i.e. $J_{i}^{\sigma} \circ J_{j}^{\sigma}=\epsilon_{i j k} J_{k}^{\sigma}$. Thus $\operatorname{dim} M=4 n$. The structures $\left\{J_{1}^{\sigma}, J_{2}^{\sigma}, J_{3}^{\sigma}\right\}$ are the sections spanning the 3 -dimensional bundle $\mathcal{G}_{U} \subset \operatorname{End}(T P)$. We shall show that $\mathcal{G}_{U}$ does not depend on $\sigma$ and there exists a global bundle $\mathcal{G}$ such that bundles $\mathcal{G}_{U}$ are the restrictions of $\mathcal{G}$, i.e. $\left.\mathcal{G}\right|_{U}=\mathcal{G}_{U}$.

The group $S O(3)$ has an adjoint representation ad in the vector space $\mathfrak{s o}(3)$ defined by $a d_{g} X=g X g^{-1}$ for $X \in \mathfrak{s o}(3)$. Let us denote

$$
a d_{g}\left(E_{i}\right)=\sum_{j=1}^{3} A_{j}^{i}(g) E_{j}
$$

where $\left\{E_{1}, E_{2}, E_{3}\right\}$ is the standard basis of $\mathfrak{s o}(3)$ corresponding to the Killing fields $\xi_{1}, \xi_{2}, \xi_{3}$. Let $V$ be the 3 -dimensional vector space and $\mathcal{C}=\left(e_{1}, e_{2}, e_{3}\right)$ be a basis of $V$. Then by $a d^{\mathcal{C}}$ we shall mean the linear representation of $S O(3)$ in $V$ defined on $\mathcal{C}$ by $a d_{g}^{\mathcal{C}} e_{i}=A_{i}^{j}(g) e_{j}$. The group $G=S O(3)$ acts on $(P, g)$ from the right by the isometries $R_{g}$. We shall also write $p g$ instead of $R_{g} p$. Note that $\nabla_{\left(R_{g}\right)_{*} X}\left(R_{g}\right)_{*} Y=$ $\left(R_{g}\right)_{*}\left(\nabla_{X} Y\right)$ where $\left(\left(R_{g}\right)_{*} X\right)_{p}=d_{p g^{-1}} R_{g}\left(X_{p g^{-1}}\right)$. Let $X=\xi^{+}$be the fundamental Killing vector field corresponding via the action of $G$ to the vector $\xi \in \mathfrak{s o}(3)$. Let us write $a_{t}=\exp (t \xi)$. Note that $\left(\left(R_{g}\right)_{*}(\xi)^{+}\right)_{p}=\frac{d}{d t}\left(p g^{-1} a_{t} g\right)=\frac{d}{d t}\left(p\left(a d\left(g^{-1}\right)\left(a_{t}\right)\right)\right)=$ $\left(a d\left(g^{-1}\right) \xi\right)_{p}^{+}$. It is also clear that

$$
p\left(\nabla_{X}\left(a d\left(g^{-1}\right) \xi_{i}\right)^{+}\right)=p\left(\sum_{j=1}^{3} A_{i}^{j}\left(g^{-1}\right) \nabla_{X} \xi_{j}^{+}\right) .
$$

Thus

$$
\begin{gathered}
p \phi_{i}\left(R_{g} \sigma^{*} X\right)=p\left(\sum_{j=1}^{3} A_{i}^{j}\left(g^{-1}\right) \nabla_{\sigma^{*} X} \xi_{j}^{+}\right) \\
\left.=p\left(\sum_{j=1}^{3} A_{i}^{j}\left(g^{-1}\right) \phi_{j} \sigma^{*} X\right)=\sum_{j=1}^{3} A_{i}^{j}\left(g^{-1}\right) p \phi_{j} \sigma^{*} X\right)
\end{gathered}
$$

for any section $\sigma \in \Gamma(P)$. Let $\sigma_{1}, \sigma_{2}$ be two sections of the bundle $P$, such that $U_{12}=\operatorname{dom} \sigma_{1} \cap \operatorname{dom} \sigma_{2} \neq \emptyset$. Then $\sigma_{1}=\sigma_{2} g_{12}$ where $g_{12}: U_{12} \rightarrow G$ is a transition function. From (3.3) it follows that

$$
\left(J_{1}^{\sigma_{1}}, J_{2}^{\sigma_{1}}, J_{3}^{\sigma_{1}}\right)=a d^{\mathcal{C}}\left(g_{12}^{-1}\right)\left(J_{1}^{\sigma_{2}}, J_{2}^{\sigma_{2}}, J_{3}^{\sigma_{2}}\right)
$$

where $\mathcal{C}=\left\{J_{1}^{\sigma_{2}}, J_{2}^{\sigma_{2}}, J_{3}^{\sigma_{2}}\right\}$. 
Hence there exists a global bundle $\mathcal{G} \subset \operatorname{End}(T P)$ which is locally spanned by the bases $\left\{J_{1}^{\sigma}, J_{2}^{\sigma}, J_{3}^{\sigma}\right\}$. From (3.4) it follows that the bundle $P$ is isomorphic to the $G$-principal fibre bundle associated with vector bundle $\mathcal{G}$. We also have

$$
\mathcal{G}=P \times_{S O(3)} \mathfrak{s o}(3) .
$$

Hence $M$ is an almost quaternion manifold. In particular $\operatorname{dim} M=4 n$. Let us define the metric $g_{*}$ on $M$ by the formula $g_{*}(X, Y)_{x}=g\left(X^{*}, Y^{*}\right)_{y}$ where $p(y)=x$ and $X^{*}, Y^{*}$ are horizontal lifts of the fields $X, Y \in \mathfrak{X}(M)$. Since $G$ acts by isometries it is clear that $g\left(X^{*}, Y^{*}\right)$ is constant on the fibers and the metric $g$ is well defined. From the definition of $g$ it is obvious that $p:(P, g) \rightarrow\left(M, g_{*}\right)$ is a Riemannian submersion. Note that each almost complex structure $J_{i}^{\sigma}$ is compatible with the metric $g_{*}$, i.e.

$$
g_{*}\left(X, J_{i}^{\sigma} Y\right)=-g_{*}\left(J_{i}^{\sigma} X, Y\right)
$$

which is a straightforward consequence of $(3.1)$. Thus $\left(M, g_{*}, \mathcal{G}\right)$ is an almost quaternion Hermitian manifold.

The 3 -K-contact bundle $P$ admits a natural connection form (see [BGM], p. 192)

$$
\omega=\eta_{1} E_{1}+\eta_{2} E_{2}+\eta_{3} E_{3} \in \mathcal{A}(P) \otimes \mathfrak{s o}(3) .
$$

Note that the metric on $P$ can be written as

$$
g=B(\omega, \omega)+p^{*} g_{*}
$$

where $B$ is the standard metric on $\mathfrak{s o}(3)$ inducing on $S O(3)$ the metric of constant sectional curvature equal to 1 . The horizontal and vertical subbundles $H_{\omega}, V_{\omega}$ with respect to the connection on $P$ defined by the form $\omega$ coincide with the horizontal and vertical bundles defined by us earlier. The vector bundle $\mathcal{G}$ defines a reduction of the $S O(4 n)$ bundle $S O(M)$ to the $S p(n) S p(1)$ subbundle

$$
Q=\left\{u \in S O(M): u I_{0} u^{-1} \in \mathcal{G}, u J_{0} u^{-1} \in \mathcal{G}, u K_{0} u^{-1} \in \mathcal{G}\right\}
$$

and we have the homomorphism of principal fibre bundles $F: Q \rightarrow P$ where we identify $P$ with the bundle of orthonormal bases of $\mathcal{G}$ defined by

$$
F(u)=\left(u I_{0} u^{-1}, u J_{0} u^{-1}, u K_{0} u^{-1}\right) .
$$

It is clear that the Levi-Civita connection of $M$ reduces to $Q$ if and only if the bundle $\mathcal{G}$ is parallel, i.e. if for any section $\sigma \in \Gamma(\mathcal{G})$ and any vector field $X \in \mathfrak{X}(M)$ we have $\nabla_{X} \sigma \in \Gamma(\mathcal{G})$. We shall find the conditions under which $\nabla \mathcal{G}=0$. Note that for $X, Y \in \mathfrak{X}(M)$

$$
\epsilon g_{*}\left(X, J_{i}^{\sigma} Y\right)_{x}=g\left(X^{*}, \phi_{i} Y^{*}\right)_{\sigma(x)} .
$$

Let us assume that $X, Y, Z \in \mathfrak{X}(M), \nabla_{Z} X_{x}=\nabla_{Z} Y_{x}=0$. Thus we have from (3.7)

$$
\begin{gathered}
\epsilon g_{*}\left(X, \nabla J_{i}^{\sigma}(Z, Y)=g\left(\nabla_{\sigma_{*} Z} X^{*}, \phi_{i} Y^{*}\right)\right. \\
+g\left(X^{*}, \nabla \phi_{i}\left(\sigma_{*} Z, Y^{*}\right)\right)+g\left(X^{*}, \phi_{i}\left(\nabla_{\sigma_{*}} Z Y^{*}\right)\right) .
\end{gathered}
$$

Note that $\left(\sigma_{*} Z\right)_{x}=Z_{\sigma(x)}^{*}+V_{\sigma(x)}$ where $V \in T_{\sigma(x)} P$ is a vertical vector. We can extend $V$ to a (vertical) Killing vector field $V=\omega\left(\sigma_{*} Z\right)^{+} \in \Gamma(V)$. Note that $\left(\nabla_{V} X^{*}\right)_{\sigma(x)}=\left(\nabla_{X}^{*} V\right)_{\sigma(x)}$. We also have $\nabla_{Z^{*}} X^{*} \in \Gamma(V), \nabla_{Z^{*}} Y^{*} \in \Gamma(V)$. Let us write $V=\omega\left(\sigma_{*}(Z)\right)=\alpha_{1} \xi_{1}+\alpha_{2} \xi_{2}+\alpha_{3} \xi_{3}$ where $\alpha_{i} \in \mathbb{R}$. Note that

$$
\begin{aligned}
\epsilon g_{*}\left(X, \nabla J_{i}^{\sigma}(Z, Y)\right) & =g\left(\nabla_{V} X^{*}, \phi_{i} Y^{*}\right)+g\left(X^{*}, \nabla \phi_{i}\left(\sigma_{*} Z, Y^{*}\right)\right. \\
& +g\left(X^{*}, \phi_{i}\left(\nabla_{\sigma_{*}} Y^{*}\right)\right) .
\end{aligned}
$$


We also have

$$
\begin{gathered}
g\left(\nabla_{V} X^{*}, \phi_{i} Y^{*}\right)+g\left(X^{*}, \phi_{i}\left(\nabla_{\sigma_{*} Z} Y^{*}\right)\right. \\
=\sum_{j=1}^{3} \alpha_{j}\left(g\left(\phi_{j}\left(X^{*}\right), \phi_{i}\left(Y^{*}\right)\right)-g\left(\phi_{i}\left(X^{*}\right), \phi_{j}\left(Y^{*}\right)\right)\right) \\
=\sum_{j \neq i} 2 \alpha_{j} g\left(X^{*}, \phi_{i} \circ \phi_{j}\left(Y^{*}\right)\right)=2 \sum_{j \neq i} \alpha_{j} \epsilon \epsilon_{i j k} g\left(X^{*}, \phi_{k}\left(Y^{*}\right)\right) .
\end{gathered}
$$

It follows that

$$
\epsilon g_{*}\left(X, \nabla J_{i}^{\sigma}(Z, Y)\right)=2 \sum_{j \neq i} \alpha_{j} \epsilon \epsilon_{i j k} g\left(X^{*}, \phi_{k}\left(Y^{*}\right)\right)+g\left(X^{*}, \nabla \phi_{i}\left(\sigma_{*} Z, Y^{*}\right)\right) .
$$

Consequently

$$
g_{*}\left(X, \epsilon \nabla J_{i}^{\sigma}(Z, Y)-2 \sum_{j \neq i} \alpha_{j} \epsilon_{i j k} J_{k}^{\sigma}(Y)\right)=g\left(X^{*}, \nabla \phi_{i}\left(\sigma_{*} Z, Y^{*}\right)\right) .
$$

Hence we have proved:

Proposition 1. Let $P$ be a 3-K-contact principal fibre bundle. Then the following conditions are equivalent:

(a) $\nabla \phi_{i}(X, Y) \in \Gamma(V)$ for $i \in\{1,2,3\}$ and any $X \in \mathfrak{X}(P), Y \in \Gamma(H)$,

(b) $R\left(X, \xi_{i}\right) Y \in \Gamma(V)$ for $i \in\{1,2,3\}$ and any $X \in \mathfrak{X}(P), Y \in \Gamma(H)$,

(c) $R(X, Y) \xi_{i}=0$ for $i \in\{1,2,3\}$ and any $X, Y \in \Gamma(H)$,

(d) $R(X, Y) Z \in \Gamma(H)$ for any $Z \in \mathfrak{X}(P)$ and $X, Y \in \Gamma(H)$.

Each of these conditions implies the following condition:

(e) the bundle $\mathcal{G}$ is parallel.

Proof. It follows from (3.10), the equality $R\left(X, \xi_{i}\right) Y=\nabla \phi_{i}(X, Y)$ and the properties of the Riemannian curvature tensor $R$.

Remark. If the 3 -K-contact structure is Sasakian, then for $X \in \mathfrak{X}(P)$ and $Y \in$ $\mathfrak{X}(M)$

$$
\nabla \phi_{i}\left(X, Y^{*}\right)=\eta_{i}\left(Y^{*}\right) X-g\left(X, Y^{*}\right) \xi_{i}=-g\left(X, Y^{*}\right) \xi_{i} \in \Gamma(V)
$$

and the condition (a) is satisfied. Hence $\left(M, g_{*}\right)$ is a quaternionic-Kähler manifold if $\operatorname{dim} M=4 n>4$. We obtain in this way the result of Ishihara ([I]).

Next we shall prove the following theorem (for $n=1$ this is a result of S. Tanno [T; we include a proof of this case for the completeness):

Theorem 2. Let us assume that $\operatorname{dim} M=4 n \neq 8$. Let $(P, g)$ be an $S O(3)$ principal 3-K-contact bundle (positive or negative) over a manifold $M$. Then the metric $g$ induces a metric $g_{*}$ on $M$ such that $p:(P, g) \rightarrow\left(M, g_{*}\right)$ is a Riemannian submersion and $\left(M, g_{*}\right)$ is a (positive or negative respectively) quaternionic-Kähler manifold. The Riemannian manifold $(P, g)$ is a 3-Sasakian (hence Einstein) manifold if $\left(P, g, \xi_{i}\right)$ is a positive 3 -K-contact structure and an $\mathcal{A}$-manifold whose Ricci tensor has two constant eigenvalues $\lambda=4 n+2$ and $\mu=-4 n-14$ if $\left(P, g, \xi_{i}\right)$ is a negative 3-K-contact structure.

Proof. Let $\omega=\eta_{1} E_{1}+\eta_{2} E_{2}+\eta_{3} E_{3}$ be as above the connection form on the bundle $P$. We have

$$
D \omega=\Omega=\Omega_{1} E_{1}+\Omega_{2} E_{2}+\Omega_{3} E_{3}
$$


where $\Omega_{i}=d \eta_{i} \circ h$ and $h: T P \rightarrow H$ is the horizontal projection. Hence from the structural equation we get

$$
\Omega_{i}=d \eta_{i}-\epsilon_{j k i} \eta_{j} \wedge \eta_{k} .
$$

Let us define the 4 -form $\tilde{\Omega} \in \mathcal{A}^{4}(P)$ by the formula $\tilde{\Omega}=\Omega_{1} \wedge \Omega_{1}+\Omega_{2} \wedge \Omega_{2}+$ $\Omega_{3} \wedge \Omega_{3}$. Note that $L_{\xi_{j}} \Omega_{i}=2\left(\epsilon_{i j k} d \eta_{k}-\eta_{j} \wedge \eta_{i}\right)=2 \epsilon_{j i k} \Omega_{k}$. Hence $L_{\xi_{j}} \tilde{\Omega}=0$ for $j=1,2,3$ and the 4 -form $\tilde{\Omega} \in \mathcal{A}^{4}(P)$ is the horizontal 4-form invariant with respect to the action of $G$. Let us define for a section $\sigma \in \Gamma(P)$ the three 2-forms $\Omega_{\sigma}^{i}=g_{*}\left(J_{i}^{\sigma} X, Y\right)$. Then $2\left(p^{*} \Omega_{\sigma}^{i}\right)_{\sigma(x)}=\epsilon d \eta_{i} \circ h_{\sigma(x)}=\epsilon\left(\Omega_{i}\right)_{\sigma(x)}$ where $h$ denotes the horizontal projection and $\epsilon \in\{-1,1\}$ was defined above. Let us define the 4 -form $\Omega_{\sigma}=\Omega_{\sigma}^{1} \wedge \Omega_{\sigma}^{1}+\Omega_{\sigma}^{2} \wedge \Omega_{\sigma}^{2}+\Omega_{\sigma}^{3} \wedge \Omega_{\sigma}^{3}$. Then $\Omega_{\sigma} \in \mathcal{A}^{4}(\operatorname{dom} \sigma)$. Note that in fact $\Omega_{\sigma}$ does not depend on $\sigma$ and there exists a global 4 -form $\bar{\Omega}$ such that $\bar{\Omega}_{\mid \text {dom } \sigma}=\Omega_{\sigma}$. We also have $4 p^{*} \bar{\Omega}=\tilde{\Omega}$. Thus

$$
4 p^{*} d \bar{\Omega}=d \tilde{\Omega} .
$$

Since the form $p^{*} d \bar{\Omega}$ is horizontal it follows that the form $d \tilde{\Omega}=D \tilde{\Omega}$ is horizontal. Thus

$$
\begin{aligned}
& d \tilde{\Omega}=2\left(d \Omega_{1} \wedge \Omega_{1}+d \Omega_{2} \wedge \Omega_{2}+d \Omega_{3} \wedge \Omega_{3}\right) \\
& =2\left(D \Omega_{1} \wedge \Omega_{1}+D \Omega_{2} \wedge \Omega_{2}+D \Omega_{3} \wedge \Omega_{3}\right) .
\end{aligned}
$$

From the Bianchi identity we have $D \Omega_{i}=0$. Consequently $d \bar{\Omega}=0, d \tilde{\Omega}=0$. From the result of Swann $[\mathrm{Sw}]$ it follows that if $\operatorname{dim} M=4 n \geq 12$, then $\nabla \bar{\Omega}=0$ and thus $\left(M, g_{*}, \mathcal{G}\right)$ is a quaternionic-Kähler manifold. In [J1], [J2] we have proved that the principal $S O(3)$-bundle $P$ associated with $\mathcal{G}$ admits canonical negative 3 -K-contact structure $\left(P_{0}, g_{0}\right)$ if $\left(M, g_{*}\right)$ has negative scalar curvature and that $\left(P_{0}, g_{0}\right)$ is then an $\mathcal{A}$-manifold whose Ricci tensor has two eigenvalues $\lambda=4 n+2$ and $\mu=-4 n-14$ of multiplicity 3 and $4 n$ respectively. In the case of positive scalar curvature $\left(P_{0}, g_{0}\right)$ is a 3-Sasakian manifold (see [J1, [J2], BGM]). We shall show that if $\operatorname{dim} M=4$, then $\left(M, g_{*}\right)$ is anti-self-dual Einstein and if $n \neq 2$, then $(P, g)$ is isometric to the canonical 3 -K-contact bundle $\left(P_{0}, g_{0}\right)$. We can treat $P$ as the bundle of orthonormal frames of $\mathcal{G}$; hence $P=P_{0}$. We shall show that $\omega=\omega_{0}$ where $\omega_{0}$ is the canonical metric connection form on $P_{0}$. To this end it is enough to prove that

$$
\nabla J_{i}^{\sigma}(Z, Y)=-2 \sum \epsilon_{i j k} \sigma^{*} \omega^{j}(Z) J_{k}^{\sigma}(Y)
$$

for every local section $\sigma \in \Gamma(P)$. We first show that $\nabla \phi_{i}\left(Z^{*}, Y^{*}\right)_{p_{0}} \in \Gamma(V)$ for every $Z, Y \in \mathfrak{X}(M)$ and a point $p_{0} \in P$. We can find a section $\sigma \in \Gamma(P)$ such that $\sigma\left(x_{0}\right)=p_{0}$ and $d_{x_{0}} \sigma(U)=U_{p_{0}}^{*} \in H_{p_{0}}$ for every $U \in T_{x_{0}} M$. Since either $(M, \mathcal{G})$ is quaternionic-Kähler or $\operatorname{dim} M=4$ and $\mathcal{G}=\wedge^{+} M$ we have $\nabla J_{i}^{\sigma}(Z, Y)=$ $\sum \theta_{i}^{j}(Z) J_{j}^{\sigma}(Y)$ where $\theta_{j}^{i}=-\theta_{i}^{j}$. Hence from (3.10) it follows that

$$
g\left(X^{*}, \nabla \phi_{i}\left(Z^{*}, Y^{*}\right)\right)_{p_{0}}=\epsilon \sum g_{*}\left(X, \theta_{i}^{j}(Z) J_{j}^{\sigma}(Y)\right)_{x_{0}} .
$$

Consequently

$$
\epsilon \nabla \Omega_{i}\left(Z^{*}, X^{*}, Y^{*}\right)_{p_{0}}=\sum \theta_{i}^{j}(Z)_{x_{0}} \Omega_{j}^{\sigma}(X, Y)_{x_{0}} .
$$

Let $Z \in T_{x_{0}} M$ be any vector and let $X=I_{1} Z, Y=I_{2} Z$. Note that $\Omega_{j}^{\sigma}(Z, X)=$ $\delta_{j}^{1} g_{*}(Z, Z), \Omega_{j}^{\sigma}(Z, Y)=\delta_{j}^{2} g_{*}(Z, Z), \Omega_{j}^{\sigma}(X, Y)=\delta_{j}^{3} g_{*}(Z, Z)$. Since $D \Omega_{i}=0$ we have 
$\mathfrak{C}_{Z^{*}, X^{*}, Y^{*}} \nabla \Omega_{i}\left(Z^{*}, X^{*}, Y^{*}\right)=0$ where $\mathfrak{C}$ denotes the cyclic sum. Consequently

$$
\sum_{j} \mathfrak{C}_{Z, X, Y} \theta_{i}^{j}(Z) \Omega_{j}^{\sigma}(X, Y)=0 .
$$

Thus $\left(\theta_{i}^{3}(Z)+\theta_{i}^{1}(Y)-\theta_{i}^{2}(X)\right) g_{*}(Z, Z)=0$. Consequently we get $\theta_{1}^{3}(Z)-\theta_{1}^{2}\left(I_{1} Z\right)=$ $0, \theta_{2}^{3}(Z)+\theta_{2}^{1}\left(I_{2} Z\right)=0, \theta_{3}^{1}\left(I_{2} Z\right)-\theta_{3}^{2}\left(I_{1} Z\right)=0$. Since $Z$ was arbitrary we have $\theta_{1}^{3}(Z)=\theta_{3}^{2}\left(I_{1} I_{2} Z\right)=-\theta_{2}^{3}\left(I_{3} Z\right)=-\theta_{1}^{2}\left(I_{2} I_{3} Z\right)=-\theta_{1}^{2}\left(I_{1} Z\right)$. Thus $\left(\theta_{1}^{3}\right)_{x_{0}}=0$ and $\left(\theta_{j}^{i}\right)_{x_{0}}=0$. Since $p_{0}$ was an arbitrary point we have $\nabla \Omega_{i} \circ h=0$, which means that $\nabla \phi_{i}\left(Z^{*}, Y^{*}\right) \in \Gamma(V)$. Note that from the first Bianchi equation we obtain $(R$ being the curvature tensor of $(P, g)) R\left(\xi_{j}, \xi_{i}\right) Y^{*}+R\left(Y^{*}, \xi_{j}\right) \xi_{i}-R\left(Y^{*}, \xi_{i}\right) \xi_{j}=0$. Consequently

$$
\nabla \phi_{i}\left(\xi_{j}, Y^{*}\right)=R\left(\xi_{j}, \xi_{i}\right) Y^{*}=-R\left(Y^{*}, \xi_{j}\right) \xi_{i}+R\left(Y^{*}, \xi_{i}\right) \xi_{j} .
$$

If $\left(P, \xi_{i}\right)$ is a positive 3 -K-contact structure, then $R\left(Y^{*}, \xi_{i}\right) \xi_{j}=\eta_{j}\left(Y^{*}\right) \xi_{i}=0$, condition (a) of Proposition 1 is satisfied and consequently (3.12) holds. In the case of negative structure we have $R\left(Y^{*}, \xi_{i}\right) \xi_{j}=-2 \epsilon_{i j k} \phi_{k}\left(Y^{*}\right)$ (see [J1). Thus from (3.14) it follows that

$$
\nabla \phi_{i}\left(\xi_{j}, Y^{*}\right)=-4 \epsilon_{i j k} \phi_{k}\left(Y^{*}\right)
$$

Consequently we obtain for an arbitrary section $\sigma \in \Gamma(P)$ (where $\alpha_{j}=\sigma^{*} \omega^{j}(Z)$ )

$$
g\left(X^{*}, \nabla \phi_{i}\left(\sigma_{*} Z, Y^{*}\right)\right)=\sum \alpha_{j} g\left(X^{*}, \nabla \phi_{i}\left(\xi_{j}, Y^{*}\right)\right)=-4 \sum \epsilon_{i j k} \alpha_{j} g\left(X, J_{k}^{\sigma} Y\right)
$$

and from (3.10) it follows that $\nabla J_{i}^{\sigma}(Z, Y)=-2 \sum_{j \neq i} \alpha_{j} \epsilon_{i j k} J_{k}^{\sigma}(Y)$. Since $\alpha_{j}=$ $\sigma^{*} \omega^{j}(Z)$ again formula (3.12) is satisfied. If $n=1$, then from the Ricci identity (see [12]) we have $\mathcal{R} \Omega_{i}^{\sigma}=2 \sum_{j<k} \epsilon_{i j k}\left(d \theta_{k}^{j}-\theta_{j}^{i} \wedge \theta_{k}^{i}\right)$ where $\mathcal{R}: \wedge M \rightarrow \wedge M$ is the curvature operator. Let the section $\sigma$ be chosen as above. Since $\theta_{k}^{j}=-2 \epsilon_{i j k} \sigma^{*} \omega_{i}$ we infer that at the point $x_{0} \mathcal{R} \Omega_{i}^{\sigma}=2 \sigma^{*} d \omega_{i}=-4 \epsilon \Omega_{i}^{\sigma}$. Since $x_{0}$ was arbitrary it follows that (see GL] Definition 1.14) $\left(M, g_{*}\right)$ is anti-self-dual Einstein with non-zero scalar curvature. Thus if $n \neq 2$ the positive (negative) 3 -K-contact bundles are in one-toone correspondence with positive (negative) quaternionic-Kähler manifolds.

Remark. S. Tanno proved (Theorem B in [T]) that for the negative quaternionicKähler manifold every canonical 3 -K-contact structure is an $n S$-structure. Consequently from our Theorem 2 it follows that if $n \neq 2$, then every negative 3 -K-contact structure is in fact an $n S$-structure. Let us note that the O'Neill tensor $A$ for the Riemannian submersion $p: P \rightarrow M$ is given by the formulas (see [BGM])

$$
\text { (a) } A_{X} Y=\sum_{i=1}^{3} g\left(\phi_{i} X, Y\right) \xi_{i}, \quad \text { (b) } A_{X} \xi_{i}=\phi_{i}(X)
$$

for any horizontal vector fields $X, Y$. Since the fibers are totally geodesic the tensor $T=0$. Hence we could also directly compute the scalar curvature and the eigenvalues of the Ricci $S$ tensor using the O'Neill formulas (see [ON]) and the fact that the connection form $\omega$ is Yang-Mills. Since $\xi_{i}$ define K-contact structures it is obvious that $S_{\mid V}=(4 n+2) i d_{V}$ (each Killing vector field defining K-contact structure is an eigenfield of the Ricci tensor with constant eigenvalue $\operatorname{dim} P-1$ ). Using the formulas in [B] (9.62) and [BGM] (p.192) we can show that $S_{\mid H}=\mu i d_{H}$ where $\mu=\frac{1}{4 n}\left(-24 n+\tau_{*}\right)$ and $\tau_{*}$ is the scalar curvature of $\left(M, g_{*}\right)$. In the positive case since $P$ is Einstein it is clear that $\tau_{*}=16 n(n+2)$ (see [BGM]). However it needs some work to show that in the negative case $\tau_{*}=-16 n(n+2)$ (see [J2]). 
The general 3-K-contact structure is bundle like with respect to the vertical foliation $V$ (see [BGM] $)$. From the general results concerning bundle like manifolds it follows that on an open and dense subset $U \subset P$ the manifold $P_{\mid U}$ is the bundle associated with the $G$-principal bundle $\tilde{P}$ with the fibre $F=G / \Gamma$ where $\Gamma$ is the discrete (hence finite) subgroup of $G$ and $G=S p(1)$ or $G=S O(3)$. Note also that if $G=S p(1)$, then $Q=\tilde{P} / \mathbb{Z}_{2}$ is an $S O(3)$-principal fibre bundle. The metric $g$ induces on $Q$ the metric $\bar{g}$ such that the natural projection $\pi: \tilde{P} \rightarrow Q$ is a local isometry. Thus the above results concerning the 3 -K-contact $S O(3)$-bundle remain valid for the $S p(1)$-principal 3-K-contact bundle. If $\operatorname{dim} P>11$, then it follows from Theorem 2 and [J2] that the positive 3-K-contact structure is then the 3-Sasakian structure. From [BGM] it is clear that in this case $P$ is an orbifold bundle over quaternionic-Kähler orbifold of positive scalar curvature $16 n(n+2)$. Analogously as in [BGM] (p.192) we have taken account of Theorem 2 and the results from [J1, JJ2]:

Theorem 3. Let $\left(P, g, \xi_{i}\right)$ be a (positive or negative) 3-K-contact manifold. Then $\operatorname{dim} P=4 n+3$. Let us assume that $P$ is complete and $n \neq 2$. If $P$ is a positive 3 -K-contact structure, then $\left(P, g, \xi_{i}\right)$ is a 3-Sasakian structure. If $P$ is a negative 3-K-contact structure, then

(a) $\left(P, g, \xi_{i}\right)$ is an $\mathcal{A}$-manifold of negative scalar curvature which is equal to $-4 n(4 n+11)+6$,

(b) the Ricci tensor $S$ of $P$ has two constant eigenvalues $\lambda=4 n+2$ and $\mu=$ $-4 n-14$ of multiplicity 3 and $4 n$ respectively,

(c) the eigendistributions of the Ricci tensor $S$ are $D_{\lambda}=\operatorname{ker}(S-\lambda I d)=V$ and $D_{\mu}=\operatorname{ker}(S-\mu I d)=H$, $\xi_{i}$,

(d) the metric $g$ is bundle like with respect to the foliation $V$ defined by the fields

(e) each leaf of the foliation $V$ is a 3-dimensional homogeneous spherical space form,

(f) the space of leaves $P / V$ is a quaternionic-Kähler orbifold of dimension $4 n$ with negative scalar curvature equal to $-16 n(n+2)$.

Remark. Note that we do not know whether Theorems 2 and 3 are true for $n=2$. Any counterexample to Theorem 2 with $n=2$ will also be an example of an almost quaternion-Hermitian manifold with closed and non-parallel fundamental 4-form $\Omega$. (See $[\mathrm{Sw}]$ for a discussion of the problem of whether $d \Omega=0$ implies $\nabla \Omega=0$ also in the case $n=2$.)

\section{ACKNOWLEDGMENTS}

This work was supported by KBN grant 2 P0 3A 01615.

\section{REFERENCES}

[B] A. Besse, Einstein Manifolds, Springer-Verlag Berlin, Heidelberg (1987). MR 88f:53087

[BGM] C.P. Boyer, K. Galicki, B.M. Mann, The geometry and topology of 3-Sasakian manifolds, J. reine. angew. Math 445 (1994), 183-220. MR 96e:53057

[GL] K. Galicki and H.B. Lawson, Quaternionic reduction and Quaternionic Orbifolds, Math. Ann 282 (1988), 1-21. MR 89m:53075

[G] A. Gray, Einstein like manifolds which are not Einstein, Geom. Dedicata. 7 (1978), 259280. MR 80b:53034

[I1] S. Ishihara, Quaternion Kählerian manifolds and fibered Riemannian spaces with Sasakian 3-structure, Kodai Math. Sem. Rep. 25 (1973), 321-329. MR 48:2943 
[I2] S. Ishihara, Quaternion Kählerian manifolds, J. Differential Geom. 9 (1974), 483-500. MR 50:1184

[J1] W. Jelonek, Almost Kähler A-structures on twistor bundles, Ann. of Global Analysis and Geometry 17 (1999), 329-339. CMP 99:17

[J2] W. Jelonek, Quaternion Kähler geometry and almost Kähler $\mathcal{A}$-manifolds, (to appear in Ann. Polon. Math.).

[K] M. Konishi, On manifolds with Sasakian 3 - structures over quaternionic Kähler manifolds, Kodai Math. Sem. Rep. 26 (1975), 194-200. MR 51:13951

[Ku] Y-Y. Kuo, On almost contact 3-structures, Tohoku Math. J. 22 (1970), 325-332. MR 43:3956

[ON] B. O'Neill, The fundamental equations of a submersion, Mich. Math. J. 13 (1966), 459469. MR 34:751

[S] S. Salamon, Quaternionic Kähler Manifolds, Inv. Math. 67 (1982), 143-171. MR 83k:53054

[Sw] A. Swann, Hyperkähler and Quaternionic Kähler geometry, Math. Ann. 289 (1991), 421450. MR 92c:53030

[T] S. Tanno, Remarks on a triple of K-contact structures, Tôhoku Math J. 48 (1996), 519531. MR 97i:53055

Institute of Mathematics, Cracow University of Technology, Warszawska 24, 31-155 Kraków, Poland

E-mail address: wjelon@usk.pk.edu.pl 